\title{
Modal Analysis Based Stress Estimation for Structural Elements Subjected to Operational Dynamic Loadings
}

\author{
Pelayo Fernández ${ }^{* 1}$, Anders Skafte ${ }^{2}$, Manuel L. Aenlle ${ }^{1}$ and Rune Brincker ${ }^{3}$ \\ ${ }^{1}$ Department of Construction and Manufacturing Engineering, \\ University of Oviedo, Gijón, Spain. \\ *fernandezpelayo@uniovi.es, aenlle@uniovi.es, \\ 2 Department of Engineering, Aarhus University, Denmark. \\ ask@ase.au.dk \\ 3 Center for Oil and Gas - Technical University of Denmark \\ runeb@dtu.dk
}

\begin{abstract}
:
The calculation of dynamic stresses plays a crucial role in the fatigue design and fatigue life prediction of structures. However, the uncertainty in the structural dynamic properties, i.e., stiffness, mass and damping, as well as the necessary use of simplified loading models, are the main sources of uncertainty in fatigue. In this work, a procedure for estimating dynamic stresses is proposed based on the real responses of the structure under dynamic loadings and the modal parameters of the structure, i.e., natural frequencies, damping ratios and mode shapes. The proposed methodology was validated by experimental tests in a simply-supported glass beam and a rectangular glass plate pinned-supported at 3 corners, both subject to dynamic random loads. The estimated stresses are compared with those measured with strain gages attached to some points of the structures. The estimated stresses are in good agreement with those provided by the strain gages which demonstrates that the methodology can be used to estimate more accurate stress time-histories.
\end{abstract}

Keywords: experimental stresses; fatigue; Operational conditions; Strain Mode Shapes. 


\section{Introduction}

Fatigue is one of the ultimate limit states that has to be verified in structures and mechanical components. In fatigue design, remaining fatigue life and fatigue retrofitting, the main sources of uncertainty are the fatigue material characterization and the real hot spot stress time histories. Fatigue analysis can be required at the design stage, during construction or in actual use. When the structure is in service, fatigue analysis can be applied to calculate performance, to compare with earlier calculations or to make decisions about the serviceability of the structure (fatigue retrofitting, out of service). Planning, testing and evaluation of fatigue data is a recursive subject appearing in the existing literature on which laboratories and research groups dealing with fatigue design are interested on.

As regarding the fatigue material characterization, no specific model seems to be generally accepted by the community, although the S-N field and the Miner Rule finds good acceptance both by the academic milieu as well as by research groups concerned with industrial applications $[1,2]$.

With respect to the stress time-histories, the uncertainty is considerably high because the results are mainly influenced by inaccuracies of the analytical or numerical model of the structure and by the use of simplified load models which does not represent, with the needed accuracy, the load characteristics (variable amplitude, random nature, frequency bandwidth, sequence effect, etc.) A numerical model of the structure, e.g. a Finite Element Model (FE;), is generally used to estimate the stresses but the stiffness, mass and damping properties of the structure can only be estimated in an approximate way. With respect to the loading, the real loads acting on structures such as wind, traffic, sea waves, etc., usually excites several modes of the structure so a broad band frequency fatigue process occurs and the simplified loading models commonly used are not 
able to reproduce with accuracy the real loading [3, 4, 5]. Moreover, sometimes the load spectrum is changed after a number of years by a modified use of the structure, which differs from the initial expectations [6] and the load spectrum must be evaluated again.

Since the main difficulties in fatigue design are due to inaccuracies in the dynamic properties of the structure and its broad band response, it seems reasonable to combine modal analysis with analytical models in order to reduce the uncertainties in the time stress-histories of the fatigue process $[7,8,9,10]$.

Modal analysis techniques are commonly used to determine the modal parameters of a system and have been applied for many years in mechanical and civil engineering applications $[11,12$, 13]. Operational modal analysis (OMA) is now an alternative to Classical modal analysis (CMA) since in OMA the testing is normally performed by just measuring the responses under the natural or operational conditions, i.e. the structure is excited by loads such as wind loads, wave loads, traffic loads, etc., which can be considered an important advantage in medium and large structures where the use of artificial excitation devices may be expensive or impractical $[14,15$, 16].

Due to the fact that testing of the structure is carried out under its operational loads conditions, the normal service of the structure does not need to be interrupted during the data measurements so it is an adequate technique for health monitoring and, it allows as a better understanding of the random loading fatigue process and, subsequently, a reliable stresses estimation.

In this work, it is presented a methodology to estimate stresses in any arbitrary point of the structure which combines a numerical model, correlated and updated using operational modal analysis, and the experimental accelerations measured at several discrete points of the structure. 
The fundamentals of the modal based stress estimation method lie, on one hand, in the correct modal identification and expansion of the mode shapes of the structure and, on the other hand, in the measurement of the responses of the structure under the real loading conditions.

The technique can be applied to a finite time segment or it can be implemented to estimate strains in real time. The proposed methodology was validated by experimental test in a simplysupported glass beam and a rectangular glass plate pinned-supported at 3 corners. The estimated time-history stresses were compared with those measured with strain gages attached to some points of the structures. The estimated stresses are in good agreement with the experimental results which demonstrates that the methodology can be used to improve the fatigue design reliability.

\section{State of the Art}

The first studies in this field appear in the 80's when modal analysis strain techniques, i.e. using strain gage sensors, began to be used to improve or complement the knowledge about the dynamic behaviour of a structure $[17,18]$. The benefits of modal strain based methods are significant since the results can be directly related with stresses and, consequently, with the integrity evaluation of a structure subject to random dynamic loading processes such as fatigue [19].

The improvement of numerical analysis applications and codes, such as the finite element method, allowed to combine strain modal analysis with numerical models. In this way, the studies conducted by Massé and Pastorel [20] report the analysis of wind turbines blades where aerodynamic loads were, firstly, frequency decomposed and, then, applied to a numerical model to obtain the stress distributions. Okubo and Yamaguchi [21] proposed a different methodology for the estimation of the dynamic operational strains based on the use of a transformation matrix that relates strain and displacement mode shapes; identified from a previous modal analysis of 
the structure. A similar process was proposed by Gun-Myung [22] but expanding the strain estimation to un-measured points.

Although nowadays numerical models are widely used in structural calculation, analytical models can be used in some simple cases such as beams where, e.g. bending Euler-Bernoulli equations can be combined with acceleration responses in order to estimate the modal stresses or strains. In this area, Ciora [23] presented a method for the determination of pipeline dynamical stress using the signals of two accelerometers and the Euler-Bernoulli bending theory where a periodical approximation for the continuous transversal motion under vibration conditions in the pipeline was used. A similar process was proposed by Chan-Jung et al. [24] where dynamic strain responses are predicted using a method based on the classical beam theory and the measured accelerations.

At the end of the 90's, OMA adds a new dimension to the modal based stress methods since only the responses of a structure are needed to identify the modal parameters [25]. This can be considered an advantage in the stress estimation problem of random fatigue processes where, in general, the forces are unknown (wind, sea waves, traffic, temperature changes, etc.).

The studies of Graugaard et al. [26] and Hjelm et al. [27] introduced operational modal analysis in the modal stress based methods. The authors proposed a method that combines a finite element model and the modal parameters of the structure identified with OMA, to predict the stresses on a structure. This methodology was applied and validated successfully by the authors in lattice structures. In this direction, Fernández et al. [28] also presented a similar method for beam structures where the modal strain mode shapes are obtained using the Euler-Bernoulli theory and the mode shapes identified with OMA. More recently, Papadioti et al. [29] proposed a modal based method using the operational responses of the structure for estimating damage accumulation due to fatigue. The authors predicted the stress responses with a high fidelity finite element model using vibration measurements from a limited number of sensors and different 
prediction methods, i.e. Kalman filter or modal expansion. The proposed stress estimations methods were validated using simulated data from a chain-like spring-mass model and a smallscale model of a vehicle structure.

With respect to the measurement techniques, nowadays a great number of different sensors such as accelerometers, LASER sensors, LVDT, etc. can be used to measure the response of the structure under different loading conditions. If the modal testing is carried out with accelerometers, the sensors used for modal identification can also be utilized to measure the response. The mode shape accuracy can be improved using surface response methods such as digital image correlation [30] or laser scanner [31].

\section{Theory}

In this work, the methodology to estimate stresses is presented for beam and Kirchoff plate finite elements.

\subsection{Bending Elements Formulation}

A variety of structures working in bending conditions can be modeled with beam elements, see Fig. 1. If the Euler-Bernoulli theory is assumed, the bending moment and the curvature can be related by the equation:

$$
E I_{y} \frac{d^{2} u}{d^{2} x}=M_{y}
$$

where $E$ is the Young's modulus, $I_{y}$ is the second moment of the cross section about y axis, $u=$ $u(z)$ is the vertical displacement (see Fig. 1) and $M_{y}$ is the bending moment. As we are interested in stresses, Navier's Law can be applied and in a section located at distance $x$ the stresses can be determined by: 


$$
\sigma(x)=\frac{M_{z}}{I_{y}} z
$$

where $z$ is the distance from the neutral axis to the point of interest in the section. Eqs. (1) and (2) can be combined and the following relation between stress and curvature is obtained:

$$
\sigma(x)=-E z \frac{d^{2} u}{d^{2} x}
$$

Due to the practical difficulties to measure the real curvature, an approximation can be introduced using the finite element method, so that the displacement in any arbitrary point of the beam element, see Fig. 1, can be obtained as [32]:

$$
u(x)=\left\{N^{e}(x)\right\}\left\{u^{e}\right\}
$$

where $\left\{N^{e}(x)\right\}$ and $\left\{u^{e}\right\}$ are vectors containing the element shape functions, e.g. Hermité polynomials, and the nodal displacements corresponding to the element $e$, respectively.

Taking into account that the second derivative of Eq. (4) is the curvature, and introducing the time dependent displacement vector, $\left\{u^{e}(t)\right\}$ in Eq. (3), the following expression is obtained:

$$
\sigma(x, t)=-E\left\{N^{e "}(x)\right\}\left\{u^{e}(t)\right\} z
$$

where superscript “" "” indicates second derivative with respect to $x$. An useful consequent step is to introduce modal-superposition [33], so that the vector $\left\{u^{e}(t)\right\}$, can be expressed in terms of the mode shapes of the structure, $\left[\phi^{e}\right]$, by means of the modal coordinates, $\{q(t)\}$, as follows:

$$
\left\{u^{e}(t)\right\}=\left[\phi^{e}\right]\{q(t)\}
$$

The introduction of the the modal coordinates in the stress estimation process is of great interest because they can be used to expand the displacements to non-measured points. Finally, if Eq. (6) is substituted in Eq. (5), the expression to determinate the stress time-histories at each point of the beam element is given by: 


$$
\sigma(x, t)=-E\left\{N^{e "}(x)\right\}\left[\phi^{e}\right]\{q(t)\} z
$$

If several elements are used to model the structure, an assembly process should be used to obtain the whole structure stress distribution.

\subsection{Kirchoff Plates Formulation}

A similar methodology as that proposed for beams can be used for plates. The strains and the small transverse (out-of-plane) displacement $u(x, y)$ in a Kirchoff thin plate (see Figure 2) are related by the equation [34]:

$$
\left\{\begin{array}{c}
\epsilon_{x} \\
\epsilon_{y} \\
\gamma_{x y}
\end{array}\right\}=-z\left\{\begin{array}{c}
\frac{\partial^{2} u}{\partial x^{2}} \\
\frac{\partial^{2} u}{\partial y^{2}} \\
2 \frac{\partial^{2} u}{\partial x \partial y}
\end{array}\right\}
$$

where $\mathrm{z}$ is the distance to the neutral surface.

If a finite element model is introduced, the displacement in any arbitrary point of a plate element is given by [34]:

$$
u(x, y)=\left[N^{e}(x, y)\right]\left\{u^{e}\right\}
$$

Where $\left[\mathrm{N}^{e}(x, y)\right]$ is a matrix containing the shape functions and $\left\{u^{e}\right\}$ is a vector containing the displacements and rotations of the nodes $\left(u_{z}, \theta_{x}, \theta_{y}\right)$. If expression (10) is now substituted in Eq. (9), the strain vector in the element is given by:

$$
\left\{\begin{array}{c}
\epsilon_{x}(x, y) \\
\epsilon_{y}(x, y) \\
\gamma_{x y}(x, y)
\end{array}\right\}=-z\left[B^{e}(x, y)\right]\left\{u^{e}\right\}
$$

Where $\left[B^{e}(x, y)\right]$ is the strain matrix of the element which contains the second derivatives of the shape functions, i.e.: 


$$
\left[B^{e}(x, y)\right]=\left\{\begin{array}{c}
\frac{\partial^{2}}{\partial x^{2}} \\
\frac{\partial^{2}}{\partial y^{2}} \\
2 \frac{\partial^{2}}{\partial x \partial y}
\end{array}\right\}\left[N^{e}(x, y)\right]
$$

Now, if modal-superposition is considered, the strains of the plate element can be expressed as:

$$
\left\{\begin{array}{c}
\epsilon_{x}(x, y, t) \\
\epsilon_{y}(x, y, t) \\
\gamma_{x y}(x, y, t)
\end{array}\right\}=-z\left[B^{e}(x, y)\right]\left[\phi^{e}\right]\{q(t)\}
$$

where the product $[B]\left[\phi_{e}\right]$ represents the curvatures of the mode shapes. Finally, the stresses at any point of the plate element can be obtained by:

$$
\left\{\begin{array}{c}
\sigma_{x}(x, y, t) \\
\sigma_{y}(x, y, t) \\
\tau_{x y}(x, y, t)
\end{array}\right\}=-z[D]\left[B^{e}(x, y)\right]\left[\phi^{e}\right]\{q(t)\}
$$

where $D$ is the constitutive matrix that depends on the material properties, which for an isotropic material is given by:

$$
[D]=\frac{E}{1-v^{2}}\left[\begin{array}{ccc}
1 & v & 0 \\
v & 1 & 0 \\
0 & 0 & \frac{1-v}{2}
\end{array}\right]
$$

with $E$ and $v$ being the Young's modulus and the Poisson ratio, respectively.

\section{Methodology}

In this section we detailed the different steps needed to apply successfully the proposed stresses based method.

\subsection{Experimental Mode Shapes of the Structure}

As it was detailed in the introduction, the modal parameters of the structure that define its dynamic behaviour must be identified, specially the experimental mode shapes of the structure, 
$[\phi]_{\mathrm{ex}}$, since the responses of the structure must be decomposed using the modal-superposition technique (see Eq. (6)). Either classical modal analysis or operational modal analysis can be used to identify the modes. Taking into account that random loads, such as natural forces, e.g. wind or sea waves, are not easily measured, operational modal analysis (OMA) results in an advantageous technique to obtain the modal parameters of the structure. Frequency Domain Decomposition (FDD) [35] or Stochastic Subspace Identification (SSI) [36] techniques can be used to identify the modes. Although modal identification implies to obtain not only the mode shapes but also natural frequencies and damping ratios, it is remarkable that in this stress estimation process, these modal parameters (natural frequencies and damping ratios) are not necessary since they will be implicitly in the response of the structure by means of the modal coordinates. In any case, the identification of frequencies and damping ratios provides always relevant information about the dynamic behaviour that could be used for additional stages, e.g. finite element model up-dating or health monitoring of the structure.

\subsection{Modal Coordinates}

Once the experimental mode shapes of the structure are obtained, the modal coordinates can be calculated using modal-superposition, see Eq. (6). Since the responses of the structure are usually measured with accelerometers, the acceleration modal coordinates are obtained from

$$
\{\ddot{q}(t)\}_{e x}=[\phi]_{e x}^{-1}\{\ddot{u}\}_{e x}
$$

Where subscript "ex" indicates experimental data and $[\phi]_{\mathrm{ex}}^{-1}$ represents the inverse matrix of the experimental mode shapes. The pseudoinverse must be used if the matrix $[\phi]_{e x}$ is not square. Then, the displacement modal coordinates, $\{\mathrm{q}(\mathrm{t})\}_{\mathrm{ex}}$, are determined using time or frequency domain integration techniques [37].

Although the stress estimation method was developed in the theory section using the mass normalized mode shapes of the structure, $[\phi]$, arbitrary normalized or un-scaled mode shapes, 
$[\psi]$, can also be used without loss of accuracy. The mass normalized mode shapes $[\phi]$ and the un-scaled mode shapes $[\psi]$ are related by [38]:

$$
[\phi]=[\psi][\alpha]
$$

where $[\alpha]$ is a diagonal matrix containing the scaling factors $[39,40]$.

Now, if Eq. (16) is substituted in Eq. (6), it results in:

$$
\left\{u^{e}(t)\right\}=\left[\psi^{e}\right][\alpha]\{q(t)\}
$$

or, alternatively:

$$
\left\{u^{e}(t)\right\}=\left[\psi^{e}\right]\left\{q^{*}(t)\right\}
$$

where $\left\{\mathrm{q}^{*}(\mathrm{t})\right\}=[\alpha]\{\mathrm{q}(\mathrm{t})\}$ are called here un-scaled modal coordinates, which can be estimated by means of the expression:

$$
\left\{q^{*}(t)\right\}=\left[\psi_{e}\right]^{-1}\left\{u_{x}(t)\right\}
$$

\subsection{Mode Shapes Expansion}

The experimental mode shapes are only known at the measured DOF's, usually translational DOF's. If we want to estimate stresses in any arbitrary point of a finite element using Eqs. (7) and (13), the mode shape components at all the DOF's of the element (translations and rotations) must be known. This implies that the experimental mode shapes have to be expanded to the unmeasured DOF's. As an example, in the bending case, we can measure all or part of the DOF's corresponding to the displacements of the structure (see Fig. 1), however it is not possible (or it is very difficult) to measure the rotations. Thus, at least an expansion to the rotational DOF's will be necessary. 
The expansion process can be carried out by different ways depending on the type of the structure (beam, plate, three dimensional, etc.), the number of DOF's measured in the experiments, the type of structure as well as the complexity of the model (analytical or numerical) considered to model the structure (beam or plate elements in opposite to hexaedric elements, etc.) [12]. The following expansion techniques have been proposed in the literature:

a) Fitting of experimental mode shapes. For simple cases such as beam-like structures (or even plates), mathematical functions that represents adequately the mode shapes such as spline curves or Fourier series [41] can be used to fit the experimental mode shapes. According to Eqs. (7) and (13), the displacements and rotations at the nodes of each element are needed and they can be obtained directly from the fitted mathematical function in those necessary points. The continuity of the function and of its first derivative must be assured. An alternative consists of determining the strain mode shapes directly from the fitted function (second derivate) at the points where the stresses are going to be estimated.

In general, these methods require a regular fine measurement grid in the testing process, mainly if high order modes are going to be fitted. With this technique, the use of analytical models or the assembly of a finite element model of the structure is avoided [41].

b) Analytical mode shapes. Another way of avoiding the assembly of a finite element model is to use the mode shape equations corresponding to analytical models, which are reported in the literature for the most common one-dimensional (beams) and two-dimensional (shell-plates) under different support conditions [42], from which the strain mode shapes are derived.

c) Numerical mode shapes. A more general method consists of using a finite element model of the structure. With this technique, the numerical mode shapes are used to expand the experimental mode shapes to the un-measured DOF's. It is assumed that the experimental mode shapes, can be expressed as a linear combination of the numerical mode shapes [43]: 


$$
\left[\phi_{e x p}^{m}\right]=\left[\phi_{F E}^{m}\right] \cdot[T]
$$

where subscripts 'ex' and ' $\mathrm{FE}$ ' indicates experimental and numerical mode shapes, respectively, superscript ' $m$ ' indicates measured DOF's and $[T]$ is a transformation matrix. Due to the fact that, in general, a low number of modes are identified in the experimental model compared with those that can be extracted from the numerical model, the matrix $[T]$ has to be estimated from the limited information given by the truncated set of experimental modes by:

$$
[T]=\left[\phi_{F E}^{m}\right]^{+}\left[\phi_{e x}^{m}\right]
$$

where $\left[\phi_{F E}^{m}\right]^{+}$is the pseudo-inverse of $\left[\phi_{F E}^{m}\right]$. The pseudo-inverse can be computed by singular value decomposition or by the corresponding least square solution:

$$
\left[\phi_{F E}^{m}\right]^{+}=\left[\left[\phi_{F E}^{m}\right]^{T}\left[\phi_{F E}^{m}\right]\right]^{-1}\left[\phi_{F E}^{m}\right]
$$

Then, the experimental mode shapes can be expanded to the un-measured degrees of freedom by means of the expression:

$$
\left[\phi_{e x}^{u m}\right]=\left[\phi_{F E}^{u m}\right] \cdot[T]
$$

where superscript 'um' indicates un-measured DOF's. The same expression can be used with unscaled mode shapes resulting in a different $[\mathrm{T}]$ matrix.

\subsection{Stress Estimation}

Finally, the stresses can be estimated using equations (7) and (13) when mass scaled mode shapes are used in beam and Kirchoff plates elements, respectively. If un-scaled mode shapes are going to be used, the stresses can be determined after substituting Eq. (19) in Eqs. (7) and (13), respectively, i.e.: 


$$
\sigma(x, t)=-E\left\{N^{e "}(x)\right\}\left[\psi^{e}\right]\left\{q^{*}(t)\right\} h
$$

for bending elements and

$$
\left\{\begin{array}{c}
\sigma_{x}(x, y) \\
\sigma_{y}(x, y) \\
\tau_{x y}(x, y)
\end{array}\right\}=-z[D]\left[B^{e}(x, y)\right]\left[\psi^{e}\right]\left\{q^{*}(t)\right\}
$$

for kirchoff plate elements.

\section{Experimental program}

\subsection{Simply supported glass beam}

A monolithic glass beam with a $100 \times 6 \mathrm{~mm}$ rectangular section and $1 \mathrm{~m}$ long was tested in the lab in simply supported configuration. A Young's modulus $E=72 \mathrm{GPa}$ and a Poisson ratio $v=$ 0.22 , respectively, were considered as mechanical properties for the glass.

The experimental modal parameters were identified with operational modal analysis. The beam was excited applying many small hits along the beam with an impact hammer, random in time and space [44]. The responses were measured using seven uniformly distributed accelerometers (models: B\&K 4508B and PCB 333B32) with a sensitivity of $100 \mathrm{mV} / \mathrm{g}$ and two strain gage sensors HBM LY11-6/350 during a period of approximately 4 minutes. The test setup is shown in Figure 3 where the arrows indicate the measured direction with the accelerometers. The responses were recorded with a sampling frequency of $1632 \mathrm{~Hz}$ and using a National Instruments Compact DAQ acquisition system equipped with NI9234 and NI9236 modules.

The modal parameters were estimated with the ARTeMIS MODAL software using the frequency-domain decomposition (EFDD) [44] and the stochastic subspace iteration (SSI) [46] methods. The two techniques provide similar results, and therefore only the modal parameters estimated with the FDD technique are presented in the paper. The first five modes for the glass 
beam were considered in the analysis. The singular value decomposition (SVD) of the responses is presented in Figure 4. On the other hand, a finite element model was also assembled in ABAQUS and the beam was discretized using 8 Euler-Bernoulli beam elements (see Figure 1).

Table 1 shows the experimental natural frequencies, identified with the EFDD technique, together with those obtained from the numerical model. It can be inferred that a very good agreement exists between the experimental and the numerical natural frequencies, the error being less than 3\%. With respect to the mode shapes, the MAC (modal assurance criterion) between the experimental and numerical mode shapes is also presented in Table 1. A very good correlation exists between the numerical and experimental mode shapes, the MAC being very close to one for all the modes considered in the investigation.

Then, the experimental mode shapes were expanded to the un-measured DOF's using the numerical mode shapes extracted from the FEM and applying the methodology presented in section 4.3.c)

The stresses at the points where the strain gages were attached were estimated using the methodology described in sections 3 and 4 . In order to excite the structure, several hits were applied to the beam in random positions, using an impact hammer. In Figure 5 is shown the power spectral density (PSD) of the experimental stresses measured with strain gage 1 (g1, see Figure 3). The peaks corresponding to the first five modes identified by OMA can easily be seen in Figure 5 together with some peaks at 50, 150, 250, 350 and $450 \mathrm{~Hz}$ corresponding to electrical noise. In Figure 5 the PSD of the un-scaled displacement modal coordinates is also presented which were calculated from the experimental accelerations and the experimental mode shapes (see section 4.2). From Figure 5, it can also be inferred that the first two modes contribute the most to the stress magnitude $\left(q_{1}^{*}(\omega)\right.$ and $\left.q_{2}^{*}(\omega)\right)$. This fact is in good agreement with the stresses 
recorded with the strain gages where the higher values correspond to modes 1 and 2 (see Figure $5)$.

In Figures 6 and 7 are presented the experimental stresses (measured with strain gages) and those estimated with Eq. (24) at points 1 and 2, (strain gages 1 and 2, respectively, see Figure 3), at different time periods. In both cases, it can be seen that a good correlation is obtained, being the average error less than $8 \%$. However, there is also a contribution of the zero-load noise level in the strain gages to the errors between the measured and the estimated stresses. On the other hand, the accelerometers are also more sensitive to impacts than the strain gages (see Figures 6 and 7) and therefore there is a significant discrepancy between the estimated and the measured stresses at the beginning of the impact loading. This effect also contributes to increase the error.

The dynamic stress distribution for the entire beam can also be calculated with the proposed methodology. In Figure 7, the estimated stresses at a specific time interval for the complete glass beam are also presented. As expected from the experimental stresses and the displacement modal coordinates (Figure 5) where modes 1 and 2 are predominant, it can be observed in Figure 7 (right) how these mode shapes appear in the stress 3D profile: 2 peaks corresponding to mode shape 2 at the beginning of each impact acting simultaneously with mode shape 1 (one peak) that can be clearly seen after vanishing mode 2 (due to its higher damping).

\subsection{A glass plate}

The second experiment was performed on a rectangular monolithic glass plate measuring $1400 \mathrm{x}$ $1000 \times 10 \mathrm{~mm}$, with a Young's modulus $E=72 \mathrm{GPa}$ and a Possion ratio $v=0.22$. The glass plate was pinned supported in 3 corners, allowing only translational movement perpendicular to the plate. 13 accelerometers and 6 strain gauge where mounted to the plate. Figure 8 illustrates the test setup. 
For the experimental modal analysis, load was induced to the plate by gently tapping it with the

finger. Two persons were exciting the plate at the same time to ensure that the load was applied all over during measurements. The accelerations were measured using B\&K4508B accelerometers and strains were measured using HBM LY11-120 strain gauges. Both accelerations and strains were acquired using the same National Instruments Compact DAQ acquisition systems used for the glass beam.

A FE model was constructed in MSC Nastran consisting of 100 DOF's and 81 QUAD4 elements. Frequencies, translational and rotational mode shapes components where extracted, and the mode shapes in terms of stains were calculated using the principles described in section 3.2 in the DOF's where the strain gauges were placed.

Experimental mode shape expansion was carried out using the same procedure as described with the simply supported beam. A plot of the singular values can be seen in Figure 9. The first 8 natural frequencies and mode shapes were estimated and used as a basis for the analysis. The MAC values between experimental and FE mode shapes are presented in Table 2, as well as the differences in frequency. The results show that the mode shapes are well correlated. The frequencies, however, show a larger deviation compared with the results from the glass beam, which is mainly explained by inaccuracies in the modelling of the supports in the FE model. However, the information needed from the FE model is limited to the mode shapes

(used in the expansion of the experimental mode shapes) because the information corresponding to the natural frequencies and the damping is contained in the experimental modal coordinates. Thus, a good accuracy can be obtained in the stress estimation if a good correlation exists in the mode shapes even though there is not a good correlation in the natural frequencies. For the plate, the experimental mode shapes were expanded using the technique described in section 4.3.c. 
For the strain estimation a series of small impulses was applied to the plate using an impact hammer. Both the measured accelerations and strains were highpass filtered at $5 \mathrm{~Hz}$ to get rid of any noise at DC, and lowpass filtered at $110 \mathrm{~Hz}$ so only 8 modes were included in the response. The following analysis is based on the bandpass filtered response.

Translations were obtained by integrating the accelerations twice with respect to time and the strains where estimated using the methodology described in section 3.2. Figure 10 shows the measured (bandpass filtered) and estimated signal for strain gauge 5 (according to Figure 8). The errors for all 6 strain gauges are shown in Table 3.

The estimation shows good results with errors between $1.4 \%$ and $6.1 \%$. The results are further illustrated by comparing the auto spectral densities (see Figure 11), and rainflow flow count (Fig. 12) for strain gauge 5. At figure showing the rainflow count the error is measured as the relative error between the individual bins. Naturally we see an increase in error for the bins with large strain values, because the fewer counts increases the uncertainty in the calculation of the error.

\section{Conclusions}

The material characterization and the stress-time history are the major sources of uncertainty in fatigue. This uncertainty comes from inaccuracies in the numerical models and from the use of simplified loading models.

The purpose of this paper is to propose an efficient method for calculating stress-time histories in structures subjected to random loadings. The technique can be applied to short time periods of in real time to structures in operation.

The proposed methodology combines the mode shapes identified by modal analysis (classical or operational), expanded using a finite element model or an alternative method, and the experimental accelerations measured at several discrete points of the structure. 
The technique has been validated by experimental tests carried out on a simply supported glass beam and on a rectangular glass plate pinned at 3 corners, comparing the estimated stresses with those recorded with strain gages.

It has been demonstrated that the stresses can be estimated with an error less than $10 \%$ if an accurate modal identification is performed and a good finite element model is assembled.

\section{Acknowledgments}

The authors would like to acknowledge the financial support given by the Spanish Ministry of Science and Innovation through the project BIA 2011-28380-C02-01 and the CajAstur Fellowship-University of Oviedo 2011 program.

\section{References}

[1] Lee YL, Hathaway, Pan J, Hathaway R, Barkey M (2005) Fatigue Testing and Analysis: Theory and Practice. Elsevier Butterworth-Heinemann

[2] Dowling NE, Thangjithan S (2001) An overview and discussion of basic methodology for fatigue. Symposium on Fatigue and Fracture Mechanics, 31:3-36

[3] Bishop NWM (1999) Vibration fatigue analysis in the finite element environment. Anales de Mecánica de la Fractura, 18:8-23

[4] Gao Z, Torgeir M (2008) Frequency-domain fatigue analysis of wide-band stationary Gaussian processes using a trimodal spectral formulation. Int J Fatigue 30:1944-1955

[5] Benasciutti D, Tovo R (2007) On fatigue damage assessment in bimodal random processes. Int J Fatigue 29:232-244

[6] Schijve J (2001) Fatigue of Structures and Materials. Kluwer Academic Publishers 
[7] Townley GE, Klahs JW (1986) Using test and system dynamic analysis for component life prediction. In Proceedings of the 4th International Modal Analysis Conference (IMAC). Los Angeles CA. Vol. 1 pp 656-662

[8] Verdonck E, Snoeys R (1984) Life time prediction based on the combined use of finite element and modal analysis data. In Proceedings of the 2nd International Modal Analysis Conference (IMAC). Orlando FL. pp 572-579

[9] Liefooghe D, Sas P. (1991) Optimizing the fatigue lifetime of structural components by using dynamic analysis methods. In Proceedings of the 9th International Modal Analysis Conference (IMAC) pp 344-351

[10] Liefooghe D, Leuridan J, Van der Auweraer H (1992) Integration of structural dynamics into fatigue prediction. Noise Vib Control Word 6-8

[11] Ewins D. (1984) Modal Testing: Theory and Practice. Research Studies Press LTD. John Wiley \& Sons INC

[12] Maia N, Silva J, He J, Lieven N, Lin R-M, Skingle, G, To W, Urgueira A (1997) Theoretical and Experimental Modal Analysis. Research Studies Press Ltd

[13] Heylen W, Lammens S, Sas P (1998) Modal Analysis: Theory and Testing. Katholieke Universiteit Leuven

[14] Cantieni R. (2005) Experimental Methods Used in System Identification of Civil Engineering Structures. In Proceedings of the International Operational Modal Analysis Conference (IOMAC). Copenhagen pp 249-260

[15] Cunha A, Caetano E (2005) From input-utput to output-only modal identification of civil engineering. In Proceedings of the 1st International Operational Modal Analysis Conference (IOMAC), Copenhagen, Denmark, pp 11-27 
[16] Møller N, Brincker R. Herlufsen H, Andersen P (2001) Modal testing of mechanical structures subject to operational excitation forces. In Proceedings of the 19th International Modal Analysis Conference (IMAC). Orlando FL. pp 262-269

[17] Bernasconi 0, Ewins DJ (1989) Modal strain/stress fields. J Modal Anal 68-76

[18] Staker C. (1985) Modal analysis efficiency improved via strain frequency response functions. In Proceedings of the 3rd International Modal Analysis Conference (IMAC), Orlando FL. Vol. 1 pp 612-617

[19] Vári LM, Heyns PS (1997) Strain modal testing - a critical appraisal. Res Dev J 13(3):8390

[20] Masse B, Pastorel H (1989) Stress calculation on the Sandia 34-meter Wind turbine with the local circulation method and turbulent wind. Institut de Reserche d'Hydro-Quebec, Varennes, QC, Canada

[21] Okubo N, Yamaguchi K (1995) Prediction of dynamic strain distribution under operating conditions by use of modal analysis. In Proceedings of the 13th International Modal Analysis Conference (IMAC), vol 1 pp 91-96

[22] Lee G-M (2007) Prediction of strain responses from the measurements of displacements responses. Mechanical systems and Signal Processing 21:1143-1152

[23] Ciora Gh T (1998) Experimental method for pipeline dynamic stress estimation by vibration measurement. In Proceedings of the 16th International Modal Analysis Conference (IMAC). Santa Barbara CA. pp 457-463

[24] Chan-Jung K, Bong-Hyun L, Hyun-Cheol J, Hyeon-Ho J, Yeon June K (2012) Estimation of strain at elastic systems using acceleration response. Transactions of the Korean Society for Noise and Vibration Engineering 22:9-14 
[25] Brincker R, Ventura C, Andersen P (2003) Why output-only modal testing is a desirable tool for a wide range of practical applications. In Proceedings of the 21st International Modal Analysis Conference (IMAC). Orlando FL. paper 265.

[26] Graugaard-Jensen J, Hjlelm H, Much K (2004) Modal based fatigue estimation. M. Sc. Thesis. Aalborg University.

[27] Hjelm H, Brincker R, Graugaard-Jensen J, Munch K (2005) Determination of stress histories in structures by natural input modal analysis. In Proceedings of the 21th International Modal Analysis Conference (IMAC) Orlando FL.

[28] Fernández P, Lopéz-Aenlle M, Brincker R, Fernández-Canteli A (2009) stress estimation in structures using operational modal analysis. In Proceedings of the 3rd International Operational Modal Analysis Conference (IOMAC) Porto Novo Ancona. Paper 225.

[29] Papadioti DC, Giagopoulos D, Papadimitriou C (2014) Fatigue monitoring in metallic structures using vibration measurements. In Proceedings of the 23rd International Modal Analysis Conference (IMAC). Orlando FL. paper 329

[30] Bing P, Kermao Q, Huimin X, Anand A (2009) Two-dimensional digital image correlation for in-plane displacement and strain measurement: a review. Meas Sci Technol, 20 (17pp), doi:10.1088/0957-0233/20/6/062001.

[31] Lee HM, Park HS (2011) Gage-free stress estimation of a beam-like structure based on terrestrial laser scanning. Comput-Aided Civ Inf, 26:647-658

[32] Yang TY (1986) Finite element structural analysis. Prentice Hall PTR.

[33] Clough Ray W, Penzien J (1993) Dynamics of structures, 2nd edition, McGraw-Hill, New York.

[34] Zienkiewicz OC, Taylor RL (2005) The finite element method for solid and structural mechanics. $6^{\text {th }}$ ed. Elsevier, Oxford 
[35] Brincker R, Zhang L, Andersen P (2001) Output-only modal analysis by frequency domain decomposition. Smart Mater Struct 10:441-445

[36] Van Overschee P, De Moor B (1996) Subspace identification for linear systems: Theory, implementation, applications. Kluwer Academic Publishers

[37] Brandt A (2011) Noise and Vibration Analysis: Signal analysis and Experimental Procedures. John Willey and Sons, LTD. United Kingdom

[38] Parloo E, Verboven P, Guillaume P, Van Overmeire (2002) M. Sensitivity - based operational mode shape normalization. Mech. Systems and Signal Proc., 16:757-767

[39] Brincker, R. and Andersen, P. (2003) A way of getting scaled mode shapes in output only modal analysis. In Proceedings of the 21 st International modal analysis conference (IMAC). Santa Barbara CA.

[40] López-Aenlle M, Brincker R, Pelayo F, Canteli, A.F. (2012) On exact and approximated formulations for scaling-mode shapes in operational modal analysis by mass and stiffness change. J Sound Vib 331:622-637

[41] Pelayo, F. (2010) Scaling factors and stresses: Estimation in structural components by means of Operational Modal Analysis. Ph.D. Thesis. University of Oviedo

[42] Blevins R.D. (2001) Formulas for natural frequency and mode shape (2 ${ }^{\text {nd }}$ Edition). Krieger Publishing Company. Florida

[43] Friswell MI, Mottershead JE (1995) Finite element model updating in structural dynamics. Kluwer Academic Publishers.

[44] Pelayo F, López-Aenlle M, Brincker R, Fernández-Canteli A (2009) Artificial Excitation in Operational Modal Analysis, In Proc. Of the 3th International Operational Modal Analysis Conference (IOMAC). Portonovo Ancona. Paper 223 
Figure Captions

Fig. 1 Example of Euler-Bernoulli beam (left) and bending element with two nodes (right)

Fig. 2 Plate element

Fig. 3 Test setup or Sensor and strain gage location for the glass beam

Fig. 4 Singular value decomposition of the experimental responses for the glass beam

Fig. 5 Power spectral density of experimental stress at point g2 (left) and displacement modal coordinates (right) for the glass beam

Fig. 6 Experimental and estimated stresses for strain gage 1 at different specific time intervals

Fig. 7 Experimental and estimated stresses for strain gage 2 (left) and estimated stress for the complete glass beam (right) at specific time intervals

Fig. 8 Sensor and strain gauge location for the rectangular glass plate

Fig. 9 Singular value decomposition of the experimental responses for the glass plate

Fig. 10 Measured and estimated strains for the glass plate

Fig. 11 Auto spectral density for measured and estimated strains at SG5 for the glass plate

Fig. 12 Comparison between measured and estimated strain in form of rainflow count (SG5) for the glass plate 
Figure1

Click here to download Figure: Figure1.docx

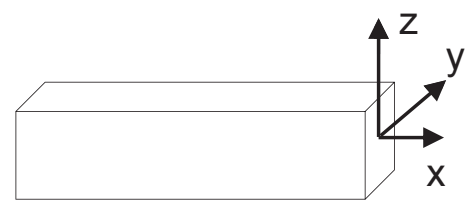

$\overbrace{1}^{4}$ 
Figure2

Click here to download Figure: Figure2.eps 


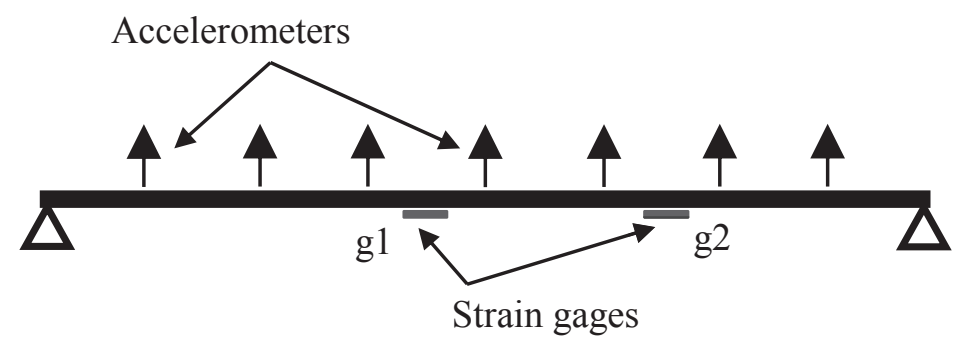

Figure3
Click here to download Figure: Figure3.docx 
Figure4

Click here to download Figure: Figure4.eps 
Figure5 (left)

Click here to download Figure: Figure5L.eps 
Figure5 (right)

Click here to download Figure: Figure5R.eps 
Figure6 (right)

Click here to download Figure: Figure6R.eps 
Figure7 (left)

Click here to download Figure: Figure7L.eps 
Figure7 (right)

Click here to download Figure: Figure7R.eps 


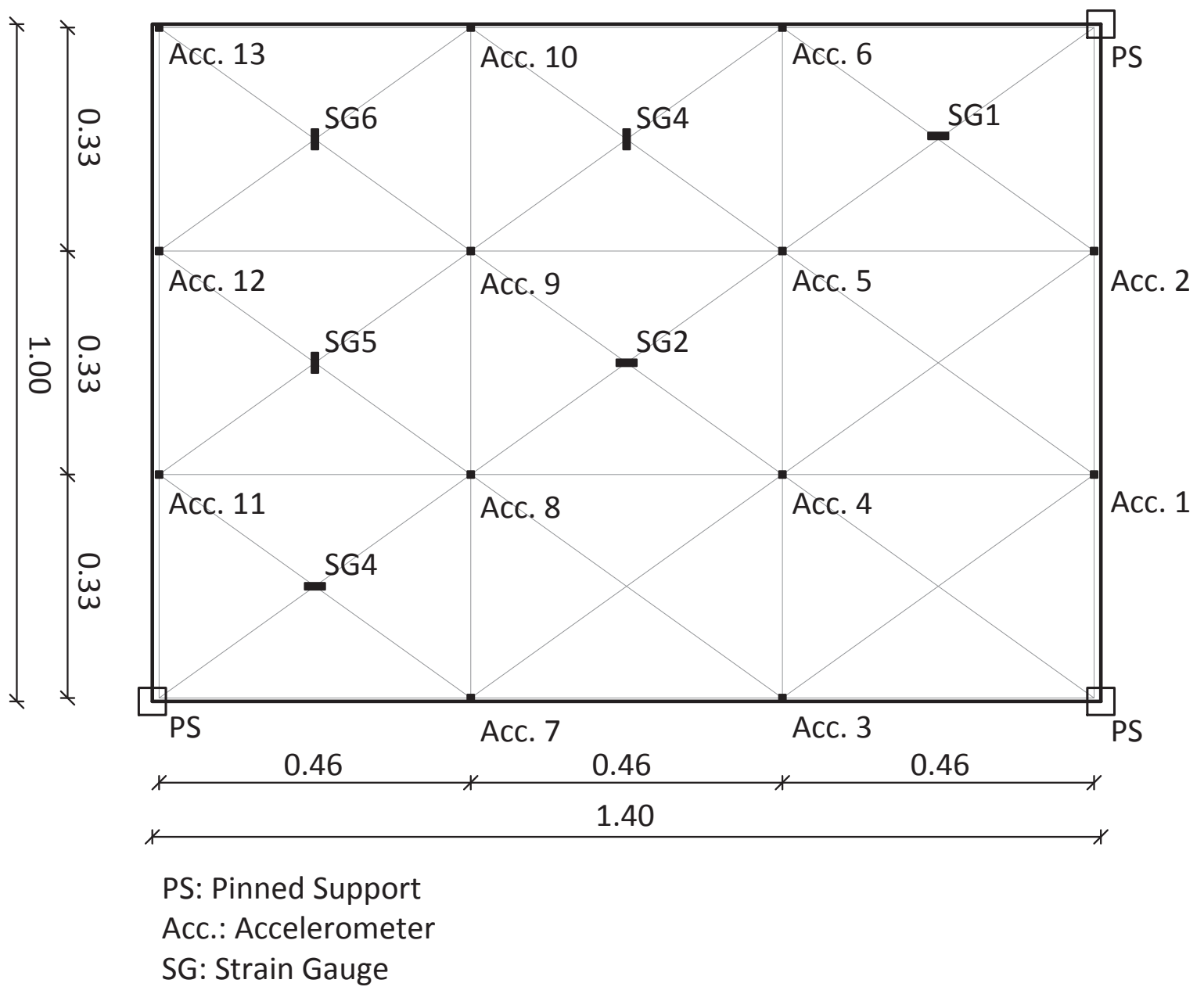


Figure9

Click here to download Figure: Figure9.eps 


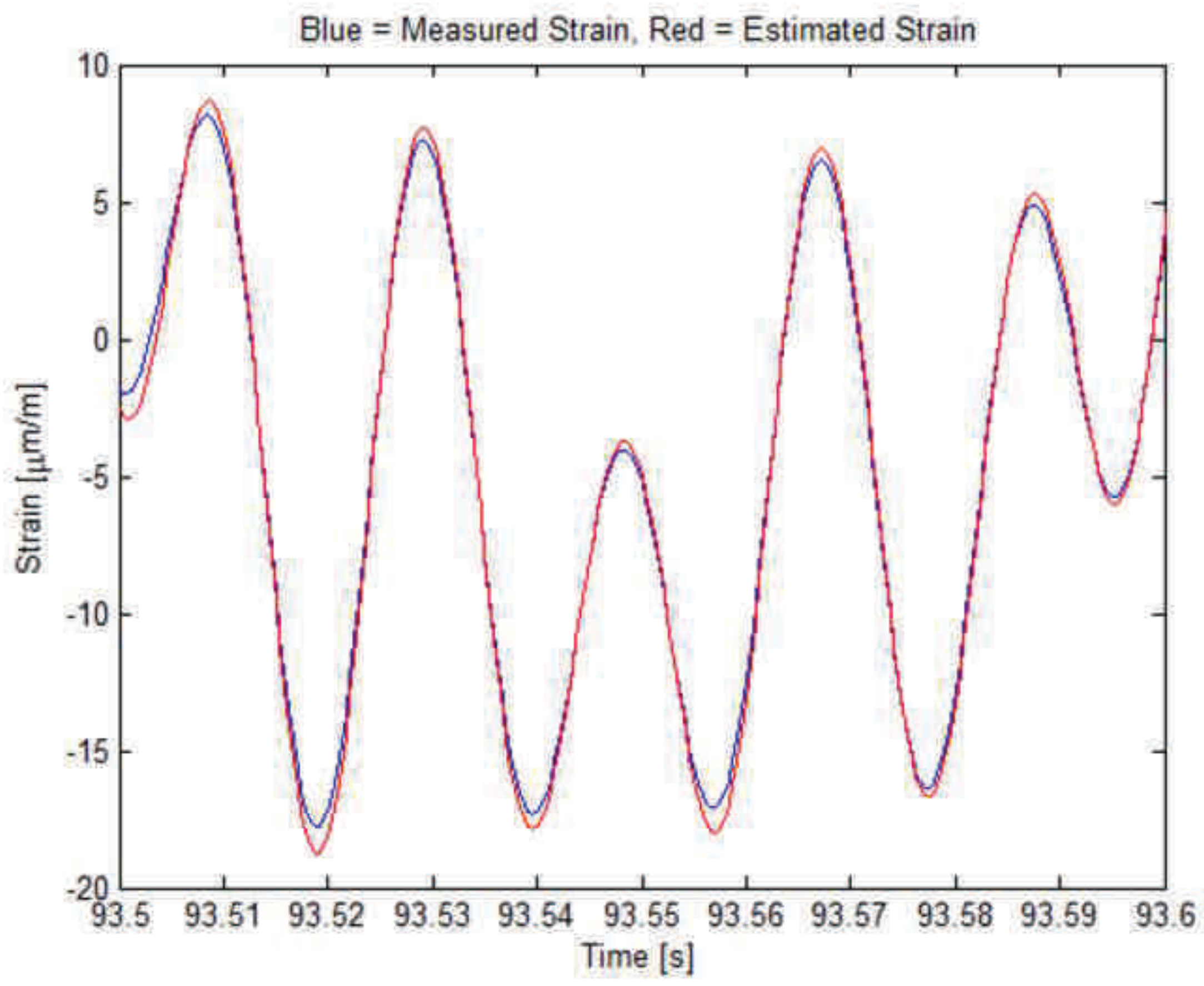







Figure11

Click here to download Figure: Figure11.eps 
Figure12

Click here to download Figure: Figure12.eps 
Figure6 (left)

Click here to download Figure: Figure6L.eps 
Table 1 Experimental and Numerical natural frequencies for the glass beam

\begin{tabular}{ccccc}
\hline & \multicolumn{3}{c}{ Frequency } & \\
\cline { 2 - 4 } Mode & $\begin{array}{c}\text { Experimetal } \\
{[\mathrm{Hz}]}\end{array}$ & $\begin{array}{c}\text { Numerical } \\
{[\mathrm{Hz}]}\end{array}$ & $\begin{array}{c}\text { Error } \\
{[\%]}\end{array}$ & \\
\hline 1 & 15.26 & 14.89 & 2.42 & 0.9999 \\
2 & 59.11 & 58.58 & 0.90 & 0.9998 \\
3 & 132.3 & 134.1 & 1.36 & 0.9995 \\
4 & 233.4 & 238.7 & 2.27 & 0.9999 \\
5 & 363.3 & 373.9 & 2.92 & 0.9988 \\
\hline
\end{tabular}


Table 2 Experimental and Numerical natural frequencies for the rectangular glass plate

\begin{tabular}{ccccc}
\hline & \multicolumn{3}{c}{ Frequency } & \\
\cline { 2 - 4 } Mode & $\begin{array}{c}\text { Experimetal } \\
{[\mathrm{Hz}]}\end{array}$ & $\begin{array}{c}\text { Numerical } \\
{[\mathrm{Hz}]}\end{array}$ & $\begin{array}{c}\text { Error } \\
{[\%]}\end{array}$ & \\
\hline 1 & 6.44 & 5.97 & 7.30 & 0.9999 \\
2 & 16.41 & 15.94 & 2.86 & 0.9994 \\
3 & 29.30 & 28.18 & 3.82 & 0.9989 \\
4 & 38.67 & 36.92 & 4.53 & 0.9987 \\
5 & 52.15 & 49.91 & 4.30 & 0.9982 \\
6 & 73.83 & 69.39 & 6.01 & 0.9980 \\
7 & 76.76 & 73.09 & 4.78 & 0.9971 \\
8 & 94.92 & 89.23 & 6.00 & 0.9971 \\
\hline
\end{tabular}


Table 3 Error between measured and estimated strains for the glass plate

\begin{tabular}{ccccccc}
\hline $\begin{array}{c}\text { Strain } \\
\text { gage }\end{array}$ & SG1 & SG2 & SG3 & SG4 & SG5 & SG6 \\
\hline Error $[\%]$ & 6.05 & 3.18 & 2.26 & 5.14 & 2.58 & 1.37 \\
\hline
\end{tabular}

